\title{
Co-administration of aspirin and adipose-derive
stem cell conditioned medium improves the functional recovery of the optic pathway in a lysolecithin-induced demyelination model
}

This article was published in the following Dove Press journal:

Neuropsychiatric Disease and Treatment

\author{
Adel Galeshi ${ }^{1,2}$ \\ Maryam Ghasemi- \\ Kasman (iD) 3,4 \\ Farideh Feizi (iD ${ }^{2,3}$ \\ Nahid Davoodian ${ }^{5,6}$ \\ Leila Zare ${ }^{7}$ \\ Zeinab Abedian ${ }^{3}$
}

'Babol University of Medical Sciences, Babol, Iran; ${ }^{2}$ Department of Anatomical Sciences, Faculty of Medicine, Babol University of Medical Sciences, Babol, Iran; ${ }^{3}$ Cellular and Molecular Biology Research Center, Health Research Institute, Babol University of Medical Sciences, Babol, Iran; ${ }^{4}$ Neuroscience Research Center, Health Research Institute, Babol University of Medical Sciences, Babol, Iran; ${ }^{5}$ Molecular Medicine Research Center, Hormozgan Health Institute, Hormozgan University of Medical Sciences, Bandar Abbas, Iran; ${ }^{6}$ Department of Clinical Biochemistry, Faculty of Medicine, Hormozgan University of Medical Sciences, Bandar Abbas, Iran; ${ }^{7}$ Department of Physiology, School of Medical Sciences, Tarbiat Modares University, Tehran, Iran

Correspondence: Maryam GhasemiKasman

Health Research Institute, Babol

University of Medical Sciences, PO Box

4136747 I76, Babol, Iran

Tel +98 II3 2190557

Fax +98 II3 2190557

Emailm.ghasemi@mubabol.ac.ir

Farideh Feizi

Department of Anatomical Sciences, Faculty of Medicine, Babol University of Medical Sciences, PO Box 4/36747/76,

Babol, Iran

Tel +98 II 32192033

$\mathrm{Fax}+98 \quad 1132199936$

Email faridehfeizi@yahoo.com
Introduction: Based on beneficial effects of aspirin and mesenchymal stem cells (MSCs) on myelin repair, in a preset study, effects of co-administration of aspirin and conditioned medium from adipose tissue-derived stem cells (ADSC-CM) on functional recovery of optic pathway, demyelination levels, and astrocytes' activation were evaluated in a lysolecithin (LPC)-induced demyelination model of optic chiasm.

Methods: LPC $(1 \%, 2 \mu \mathrm{L})$ was injected into the rat optic chiasm and animals underwent daily intraperitoneal (i.p.) injections of ADSCs-CM and oral gavage of aspirin at a dose of 25 $\mathrm{mg} / \mathrm{kg}$ for 14 days post LPC injection. The conductivity of visual signals was assessed using visual evoked potential recordings (VEPs) before LPC injection and on days 7 and 14 post lesion. Immunostaining against PDGFR $\alpha$ as oligodendrocyte precursor cells marker, MOG as mature myelin marker, and GFAP as astrocyte marker was performed on brain sections at day 14 post LPC injection. FluoroMyelin staining was also used to measure the extent of demyelination areas.

Results: Our results showed that administration of ADSCs-CM and aspirin significantly reduced the latency of VEP waves in LPC receiving animals. In addition, demyelination levels and GFAP expressing cells were attenuated while the number of oligodendrocyte precursor cells significantly increased in rats treated with ADSCs-CM and aspirin.

Conclusion: Overall, our results suggest that co-administration of ADSCs-CM and aspirin improves the functional recovery of optic pathway through amelioration of astrocyte activation and attenuation of demyelination level.

Keywords: lysolecithin, demyelination, optic chiasm, mesenchymal stem cells, conditioned medium, aspirin

\section{Introduction}

Multiple sclerosis (MS) is regarded as the most common cause of neurological disability of young adults worldwide. ${ }^{1,2}$ However, the etiology of MS has not been fully understood, but it is postulated that MS is a chronic autoimmune inflammatory disease of the central nervous system (CNS). ${ }^{3}$ During MS disease, over-activation of inflammatory $\mathrm{T}$ cells leads to autoimmune attacks and subsequent myelin damage and axonal loss. ${ }^{3}$ Available therapies for MS mainly target the immune system and there are no effective medications for enhancement of myelin repair. ${ }^{2,4}$ Oligodendrocytes precursor cells (OPCs) are considered as the main cellular source 
for generation of new myelin-forming cells in the CNS. Following demyelination, the resident OPCs are activated and their levels of proliferation, recruitment, migration, and differentiation to the mature oligodendrocytes will increase in damaged areas. ${ }^{5}$ Although, due to the limited number of endogenous OPCs, the capacity of endogenous mechanism for remyelination is low and poor. ${ }^{5,6}$ Therefore, any drug which can increase the number of OPCs and promote remyelination, is considered as a useful approach for the treatment of MS. ${ }^{7}$

Stem cell therapy has emerged as an ideal strategy for the treatment of various types of CNS-related disorders such as MS., Different types of stem cells including autologous hematopoietic, neuronal, induced pluripotent, and human embryonic stem cells have been introduced as potential therapeutic approaches in MS. ${ }^{10}$ Additionally, mesenchymal stem cells (MSCs) have emerged as the most promising stem cell type for treating MS patients. ${ }^{11}$ Because of their abundance and accessibility, adipose tissue-derived stem cells (ADSCs) have been introduced as an alternative source to bone marrow MSCs. ${ }^{12-14}$ It has been shown that MSCs have remarkable immunomodulatory properties and neuroprotective effects. ${ }^{15-17}$ In addition, it has been demonstrated that MSCs reduce the extent of demyelination areas and increase neural stem cells' (NSCs) differentiation toward myelin-forming cells. $^{18,19}$

In spite of the beneficial effects of MSCs in MS disease, there are several limitations such as HLA related incapability, tumorigenicity, and ethical concerns that have hampered the application of MSCs. ${ }^{2}$ Several lines of evidence showed that the beneficial effects of MSCs are more likely due to paracrine factors that are secreted by MSCs, not effective integration and differentiation of the cells in the damaged area. ${ }^{20,21}$ Interestingly, it has been demonstrated that adipose tissue-derived stem cells conditioned media (ADSC-CM) contains various growth factors that play important roles in tissue repair. ${ }^{22}$ Several pieces of evidence suggested that MSCs' secreted factors exert immunomodulatory and neuroprotective activities. ${ }^{5,23,24}$ It has also been illustrated that ADSCs-CM could reduce the severity of experimental autoimmune encephalomyelitis (EAE) in a model of MS. ${ }^{2}$ Furthermore, MSC-CM significantly promotes the differentiation of OPCs and enhances endogenous remyelination. ${ }^{5}$

In addition to stem cell therapy, several novel medications have also been developed to improve the potency of transplanted cells for enhancement of endogenous remyelination. A previous report by Yazdi et al, indicated that fingolimod, as an anti-inflammatory drug, increases the survival and differentiation of transplanted neural progenitors to oligodendrocyte lineage cells in a local demyelination of corpus callosum model. ${ }^{25}$

Aspirin, also known as acetylsalicylic acid (ASA), is one of the most widely used anti-inflammatory and antipyretic-analgesic drugs. ${ }^{3}$ Interestingly, aspirin has been proposed as an effective drug for treatment of MS-related fatigue. $^{26,27}$ Furthermore, it has been shown that aspirin ameliorates the disease process in MS by reducing the levels of inflammation and inhibition of mitochondrial complex I activity. ${ }^{28}$ Additionally, it has been shown that low dose of aspirin increases OPCs' proliferation, while its administration at high dose promotes OPCs' differentiation in a white matter lesion model. ${ }^{7}$ Moreover, Huang et al demonstrated that aspirin enhances OPCs' differentiation via inhibition of $\mathrm{Wnt} / \beta$-catenin signaling pathway. ${ }^{29}$ In a most recent study, Mondal et al illustrated that aspirin reduces the clinical symptoms of EAE by reducing inflammatory response and demyelination extension. ${ }^{3}$ It has also been shown that aspirin up-regulates the expression of ciliary neurotrophic factor (CNTF) in cultured astrocytes. ${ }^{30}$ CNTF is regarded as a pro-myelinating trophic factor that plays an important role in survival of oligodendrocytes as well as maturation of OPCs toward myelin-forming cells. ${ }^{31}$

Lysolecithin (LPC)-induced demyelination model has been introduced as the most common focal demyelination model for evaluation of demyelination and remyelination processes in the CNS. ${ }^{32}$ Focal injection of LPC into the white matter such as optic chiasm is regarded as a useful approach not only for studying the normal events involved in the remyelination process, but also as a preclinical tool for screening of novel remyelination-promoting drugs. ${ }^{33}$ Visual evoked potential (VEP) recording is widely used in clinical settings to assess the severity of optic neuritis in acute phase of MS disease, and also to monitor the disease course in the follow-up period. ${ }^{34}$ Interestingly, it has been shown that changes in the VEP parameters closely correlate with pathological damage in the optic nerve. ${ }^{35}$ In addition, local injection of LPC into the optic chiasm of animals and consequent assessment of visual signals' delay using VEP recording has emerged as reliable tool for evaluation of demyelination and remyelination. ${ }^{34,36}$

Based on this evidence, in the present study, the effect of aspirin and ADSCs-CM on functional recovery of optic pathway was evaluated in an LPC-induced demyelination 
model. Furthermore, the extent of demyelination areas and astrocytes' activation of the optic chiasm were assessed using immunostaining on day 14 post insult.

\section{Materials and methods Culture of ADSCs}

ADSCs were a gift from Dr. Davoodian's lab (Hormozgan University of Medical Sciences, Bandar Abbas, Iran). To isolate ADSCs, human adipose tissue was obtained from discarded lipoaspirate during liposuction surgery, with patient consent, and approval from Hormozgan University of Medical Sciences' Ethics Committee. ${ }^{37}$ Cells were cultured in culture medium (DMEM, 10\% FBS and 1\% penicillin-streptomycin) and incubated at $37{ }^{\circ} \mathrm{C}$ and $5 \% \mathrm{CO}_{2}$.

\section{Flow cytometry analysis}

In order to assess the expression of cell surface antigens, flow cytometry analysis was used based on a previous report. ${ }^{37}$ Briefly, cultured cells were detached using trypsin-EDTA and approximately $2 \times 10^{5}$ cells were labeled with FITC or PE-conjugated anti-CD90 (Dako Denmark A/S, Glostrup, Denmark), anti-CD105 (R\&D Systems, Inc., Minneapolis, MN, USA), anti-CD45 (BD PharmingenTM, USA), anti-CD73 (BD PharmingenTM), anti-CD34 (BD PharmingenTM) or mouse IgG isotype negative control antibodies (BD PharmingenTM).

\section{Adipogenic and osteogenic differentiation of ADSCs}

The ability of cultured ADSCs for differentiation to adipocyte and osteocyte was evaluated based on a previous report by Azandaryani et al. ${ }^{37}$ In brief, ADSCs were incubated with adipogenic or osteogenic differentiation culture medium for 14 days. Adipogenic and osteogenic differentiation were evaluated using Oil Red O (SigmaAldrich Co., St Louis, MO, USA) and Alizarin red S (Sigma-Aldrich Co.) staining, respectively.

\section{Preparation of conditioned medium}

Preparation of $\mathrm{CM}$ was performed based on a previous report by Yousefi et al. ${ }^{2}$ In brief, ADSCs at passage 2 (70$80 \%$ confluency) were cultured in serum-free DMEM medium for $48 \mathrm{~h}$. Culture medium was collected and centrifuged for $3 \mathrm{~min}$ at $440 \times \mathrm{g}$. Then, the upper phase was centrifuged for $3 \mathrm{~min}$ at $17,400 \times g{ }^{38}$ The resulting supernatant was collected and filtered through a $0.22 \mu \mathrm{m}$ membrane and stored at $-80^{\circ} \mathrm{C}$.

\section{Animals}

In this study, thirty adult male Wistar rats (weighting 200$250 \mathrm{~g}$ ) were obtained from Babol University of Medical Science's animal house (Babol, Iran). Animals were kept in $12 \mathrm{~h} \mathrm{light/dark} \mathrm{cycle} \mathrm{and} \mathrm{they} \mathrm{had} \mathrm{free} \mathrm{access} \mathrm{to} \mathrm{food} \mathrm{and}$ water. All experimental protocols were approved by the local ethics committee of the Babol University of Medical Sciences, which was in accordance with NIH guide for the Care and Use of Laboratory Animals.

\section{Experimental groups}

Animals were randomly divided into five groups $(\mathrm{n}=6$ in each group) as follows:

Group 1 as Saline + Saline: $2 \mu \mathrm{L}$ saline as LPC vehicle was injected into the rat's optic chiasm and then animals were treated with saline (i.p.) for 14 days post insult.

Group 2 as LPC + Vehicle: LPC $(1 \%, 2 \mu \mathrm{L})$ was injected into the optic chiasm and then rats received saline (oral) as vehicle of aspirin and culture medium (i.p.) for 14 days.

Group 3 as LPC + ADSCs-CM: LPC $(1 \%, 2 \mu \mathrm{L})$ was injected into the rat's optic chiasm and ADSCs-CM was i.p. administrated for 14 days.

Group 4 as LPC + Aspirin: $2 \mu \mathrm{L}$ of LPC (1\%) was injected into the optic chiasm and then aspirin at dose of $25 \mathrm{mg} / \mathrm{kg}^{7}$ was orally administrated for 14 days post lesion.

Group 5 as LPC + ADSCs-CM + aspirin: in this group, after injection of LPC into the optic chiasm, ADSCs-CM (i.p.) and aspirin (oral) were co-administrated for 14 days post lesion.

\section{Induction of demyelination model}

To induce demyelination model, LPC was injected into the optic chiasm based on our previous studies. ${ }^{36,39}$ In brief, animals were anesthetized with ketamine $(100 \mathrm{mg} / \mathrm{kg})$ and xylazine $(10 \mathrm{mg} / \mathrm{kg})$ and fixed in stereotaxic instrument (Stoelting, USA). LPC $(1 \%, 2 \mu \mathrm{L})$ was injected into the optic chiasm using a Hamilton syringe (Coordinates: AP and ML: 0 from bregma and $\mathrm{DV}=-7.4 \mathrm{~mm}$ from dura surface). ${ }^{36,40}$ LPC was injected within $2 \mathrm{~min}$ and the needle was kept in for an additional 3 min to avoid possible backflow through the needle tract.

\section{Visual evoked potential recording}

To assess the latency of visual signals, VEP recordings were carried out as we previously described ${ }^{34}$ In brief, two monopolar electrodes as recording (AP: $-7 \mathrm{~mm}, \mathrm{ML}:-3 \mathrm{~mm}$ ) from bregma and reference electrodes (AP: $+2 \mathrm{~mm}, \mathrm{~L}:+1.5 \mathrm{~mm}$ ) 
were implanted on the occipital and frontal lobe, respectively. Dark adaptation was performed for $10 \mathrm{~min}$ and general evoked response stimulator (D3111 Data Acquisition, ScieneBeam Co., Tehran, Iran) was used for light stimulation. To analyze the results of VEPs, the latency between the flashlight and the first negative wave (N1) was assessed using eprobe software (ScieneBeam Co., Tehran, Iran).

\section{Immunostaining}

Immunostaining procedure was done as we mentioned previously. ${ }^{41,42}$ In brief, brain samples were collected and fixed in $4 \%$ paraformaldehyde (PFA) for $12-16 \mathrm{~h}$. Coronal sections $(6 \mu \mathrm{m})$ were taken from the optic chiasm region with a cryostat apparatus (MICROM HM 525, Thermo Scientific, Germany). For immunostaining, tissue sections were washed with PBS and blocking solution including normal goat serum (NGS) $10 \%$ and triton X-100 (0.3\%) was used for blocking of non-specific bindings of antibody to antigens. Then, primary antibodies including rabbit anti-GFAP (1:400, Z0334, Dako Denmark A/S), mouse anti-MOG (1:100, MAB5680, Millipore) or mouse anti-PDGFR $\alpha$ (1:50, sc-21789, Santa Cruz Biotechnology Inc., Dallas, TX, USA) were added on the glass slides overnight at $4{ }^{\circ} \mathrm{C}$. Tissue sections were washed with PBS and incubated with secondary antibodies including Goat Anti-Rabbit conjugated Alexa Fluor ${ }^{\circledR} 594$ (1:1000, Abcam Inc, ab150077) or Goat Anti-Mouse IgG H\&L (Alexa Fluor ${ }^{\circledR}$ 594) (1:1000, Abcam Inc, ab150116) for $1 \mathrm{~h}$ at room temperature. Then, sections were washed with PBS and cell nuclei were stained using 4', 6-diamidino-2phenylindole (DAPI). Tissue sections were evaluated under Olympus IX71 microscope and images from the optic chiasm were taken by DP-27 camera (Olympus Corporation, Tokyo, Japan). Quantification of immunostaining results was performed as we mentioned in our previous studies. ${ }^{34,36,43}$ The number of GFAP or PDGR $\alpha$ positive cells were counted using Image J software (version $1.42 \mathrm{~V}$, NIH, USA). To analyze the immunostaining results, 3 sections from each slide and 3 slides from each animal and 3 rats were used for each group. ${ }^{40,41}$

\section{Histological staining}

To determine the extent of demyelination areas, FluoroMyelin staining was used as we mentioned previously. ${ }^{34}$ Demyelination levels were assessed as the percentage of total area of optic chiasm by Image $\mathrm{J}$ software (version 1.42 V, NIH, USA) based on our previous reports. ${ }^{34,36}$ Nine tissue sections from each animal and 3 animals from each group were used for histological evaluation.

\section{Statistical analysis}

Experimental results were analyzed using GraphPad Prism software (version 6.1). Electrophysiological results were analyzed by two-way ANOVA followed by Bonferroni post-test. Histological data were measured using one-way ANOVA followed by Tukey post-hoc test. The experimental results are expressed as mean \pm SEM and $P$-values $<0.05$ were considered statistically significant.

\section{Results}

\section{Characterization of ADSCs}

For characterization of ADSCs, flow cytometry analysis was used to measure the expression levels of ADSCs' specific surface markers. Our data showed that MSCs' specific markers including CD105 (98.45\%), CD90 (98.86\%), and CD73 (99\%) were highly expressed in cultured ADSCs. Interestingly, the cultured cells were negative for hematopoietic markers such as CD34 $(1.05 \%)$ and $\mathrm{CD} 45$ (1.10\%) (Figure 1A). In addition, we evaluated the potency of ADSCs for mesodermal differentiation. Results of Alizarin red staining demonstrated the osteogenic differentiation of ADSCs (Figure 1B). Oil Red O staining also confirmed the adipogenic fate of cultured MSCs compared to the undifferentiated ADSCs (Figure 1B).

\section{Co-administration of ADSCs-CM and aspirin improved the functional recovery of optic pathway}

In order to determine the effect of ADSCs-CM and aspirin on the conductivity of visual signals, VEP waves were recorded on days 0,7 , and 14 post lesions. Results of VEP recording illustrated that there was no significant difference in N1 latency between experimental groups before LPC injection. In comparison to the saline + saline group, injection of LPC significantly increased the latency of VEP waves in LPC + vehicle treated animals on days 7 and $14(P<0.001)$. There was also a significant difference in conductivity of visual signals in LPC + aspirin compared to the saline + saline on day 7 post LPC injection $(P<0.01)$. The N1 latency was significantly reduced in animals under treatment of ADSCs$\mathrm{CM}$ or aspirin or ADSCs-CM + aspirin compared to the LPC + vehicle $(P<0.001)$ on days 7 and 14 post lesion. Interestingly, co-administration of ADSCs-CM and aspirin could improve the functional recovery of the 
(A)
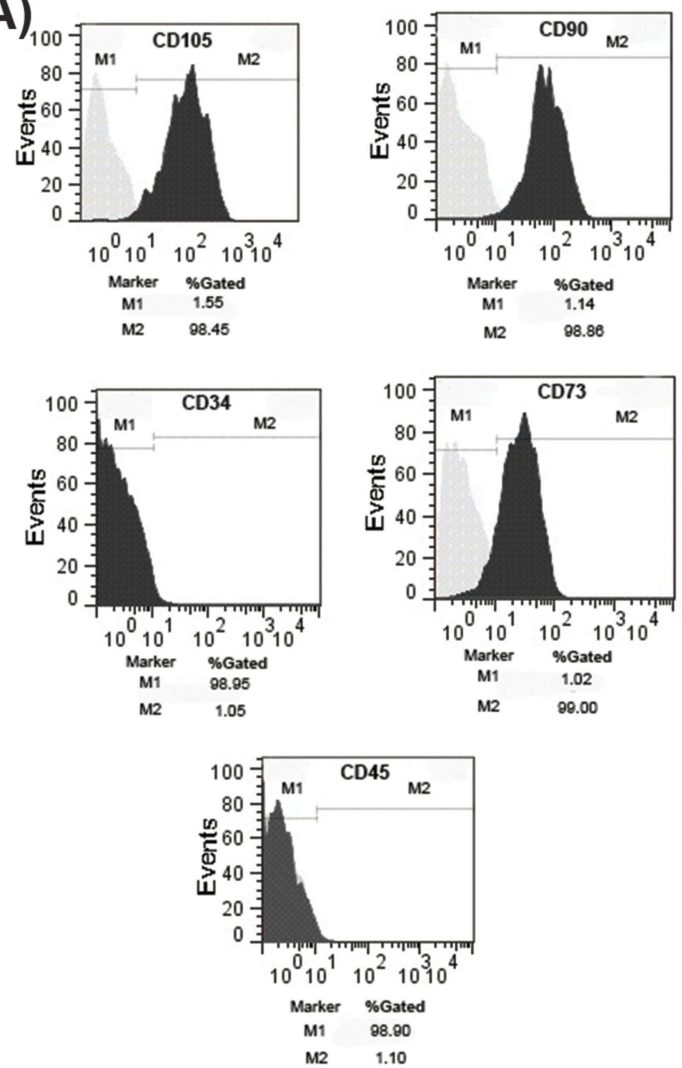

(B)
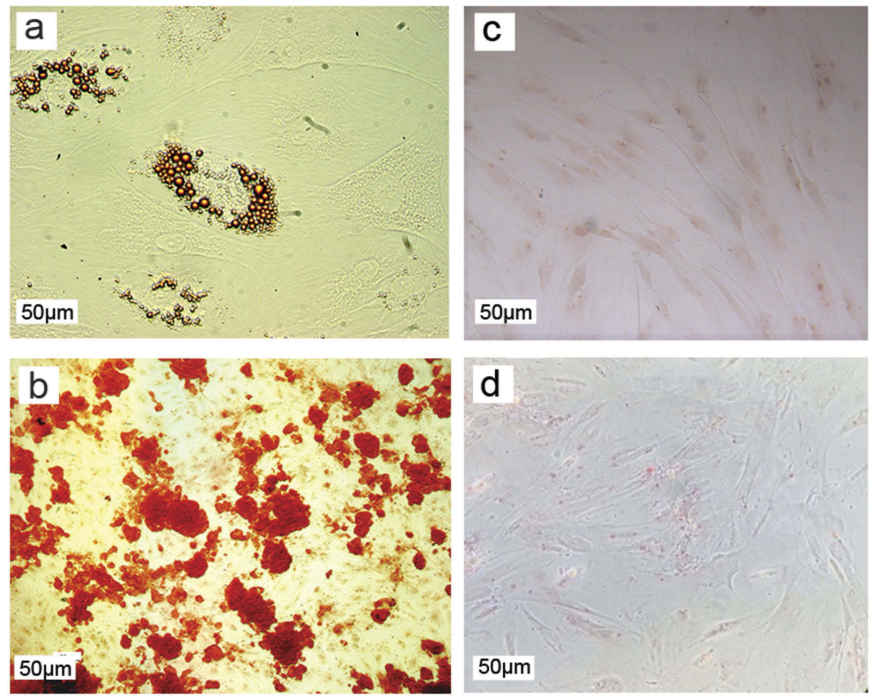

d

Figure I Characterization of cultured ADSCs. (A) Flow cytometry analysis showed that cultured cells expressed the specific markers of ADSCs such as CDI05, CD90, and CD73, while they were negative for CD34 and CD45 as hematopoietic stem cells markers. (B) Differentiation capacity of ADSCs into adipocytes (a) and osteoblasts (b); (c, d) undifferentiated cells as negative control. Scale bar: $50 \mu \mathrm{m}$.

optic pathway compared to the LPC + aspirin on days 7 $(P<0.001)$ and $14(P<0.01)$ post insult. Additionally, in comparison with LPC + ADSCs-CM, the latency of visual signals was decreased in animals treated with both ADSCs-CM and aspirin $(P<0.05)$ on day 7 post LPC injection (Figure 2A and B). These data demonstrated that co-administration of ADSCs-CM and aspirin improved the functional recovery of the optic pathway in demyelination context.

\section{Co-administration of ADSCs-CM and aspirin attenuated the extent of demyelination areas in the optic chiasm}

To evaluate the effect of ADSCs-CM and aspirin on the levels of demyelination, FluoroMyelin staining was used on day 14 post LPC injection. Our data demonstrated that ADSCs-CM or aspirin or ADSCs-CM + aspirin significantly alleviated the extent of demyelination areas compared to the LPC + vehicle $(P<0.001)$. In addition, there was a significant difference in the level of demyelination between LPC + aspirin and LPC + ADSCs-CM + aspirin treated animals $(P<0.05)$ (Figure $3 \mathrm{~A}$ and $\mathrm{B}$ ).

Additionally, immunostaining against MOG as mature myelin marker also illustrated that demyelination levels were reduced in animals receiving ADSCs-CM, aspirin or ADSCs-CM + aspirin compared to the LPC + vehicle on day 14 post insult $(P<0.001)$. In addition, co-administration of ADSCs-CM and aspirin led to stronger effect on reduction of demyelination levels compared to the LPC + ADSCs-CM $(P<0.05)$ and $\mathrm{LPC}+$ aspirin $(P<0.01)$ (Figure $4 \mathrm{~A}$ and $\mathrm{B}$ ). These findings indicated that coadministration of aspirin and ADSCs-CM attenuate the levels of demyelination in context of demyelination.

\section{Co-administration of ADSCs-CM and aspirin enhanced the number of oligodendrocyte precursor cells in the optic chiasm}

In order to determine the effect of ADSCs-CM and aspirin on the population of OPCs, immunostaining against 
A

\section{A}
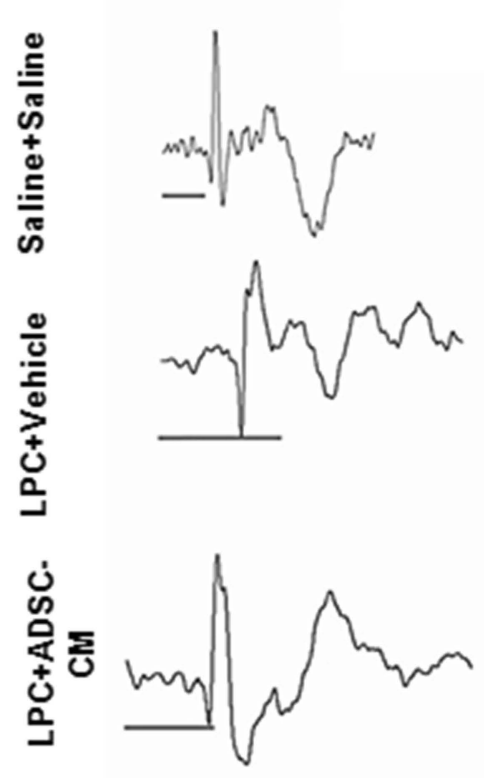

:

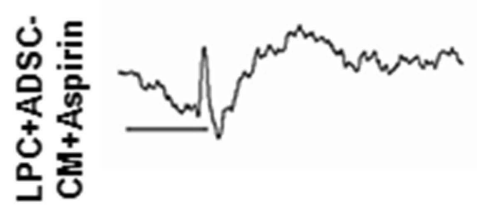

$14 \mathrm{dpi}$<smiles>CCCCC(C)CCCC</smiles>

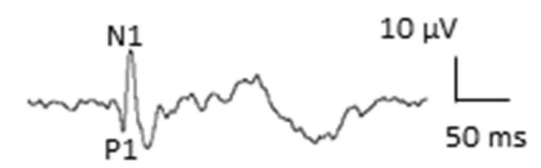

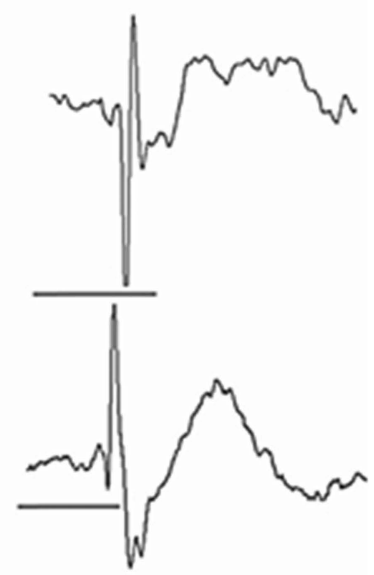

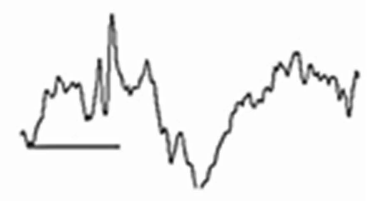

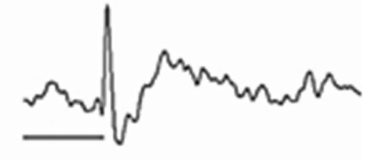

B

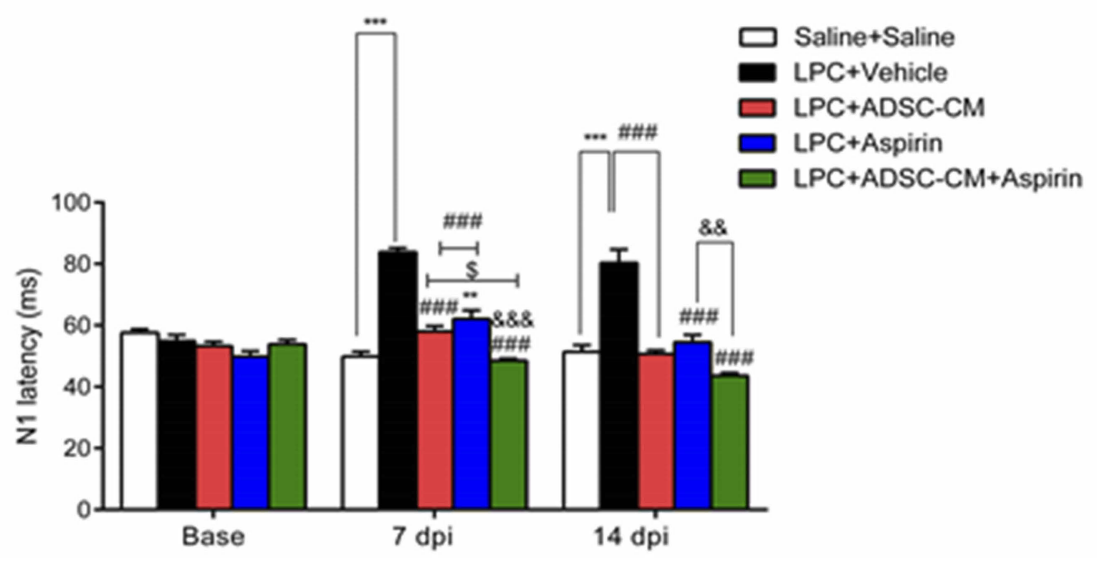

Figure 2 Effect of ADSCs-CM and aspirin on conductivity of visual signals. (A) Sample traces of VEP waves in experimental groups. Scale bar: voltage: $10 \mu \mathrm{V}$, time: 50 ms. (B) Quantification of VEP data indicated that co-administration of ADSCs-CM and aspirin improved the functional recovery of optic pathway. Two-way ANOVA followed by Bonferroni post-test were used for statistical analysis. $* * P<0.01$ and $* * * P<0.001$ compared to the saline + saline; $P<0.001$ compared to the $L P C+$ vehicle; $\& \& P<0.01$ and ${ }^{2 \& \&} P<0.001$ compared to the LPC + aspirin and ${ }^{\$} P<0.05$ compared to the LPC + ADSCs-CM. $n=6$.

Abbreviation: Dpi, days post injection.

PDGFR $\alpha$ as OPCs marker was performed on brain sections. In comparison with saline + saline, ADSCs-CM and aspirin increased the number of PDGFR $\alpha$ positive cells following LPC injection $(P<0.001)$. The number of OPCs 

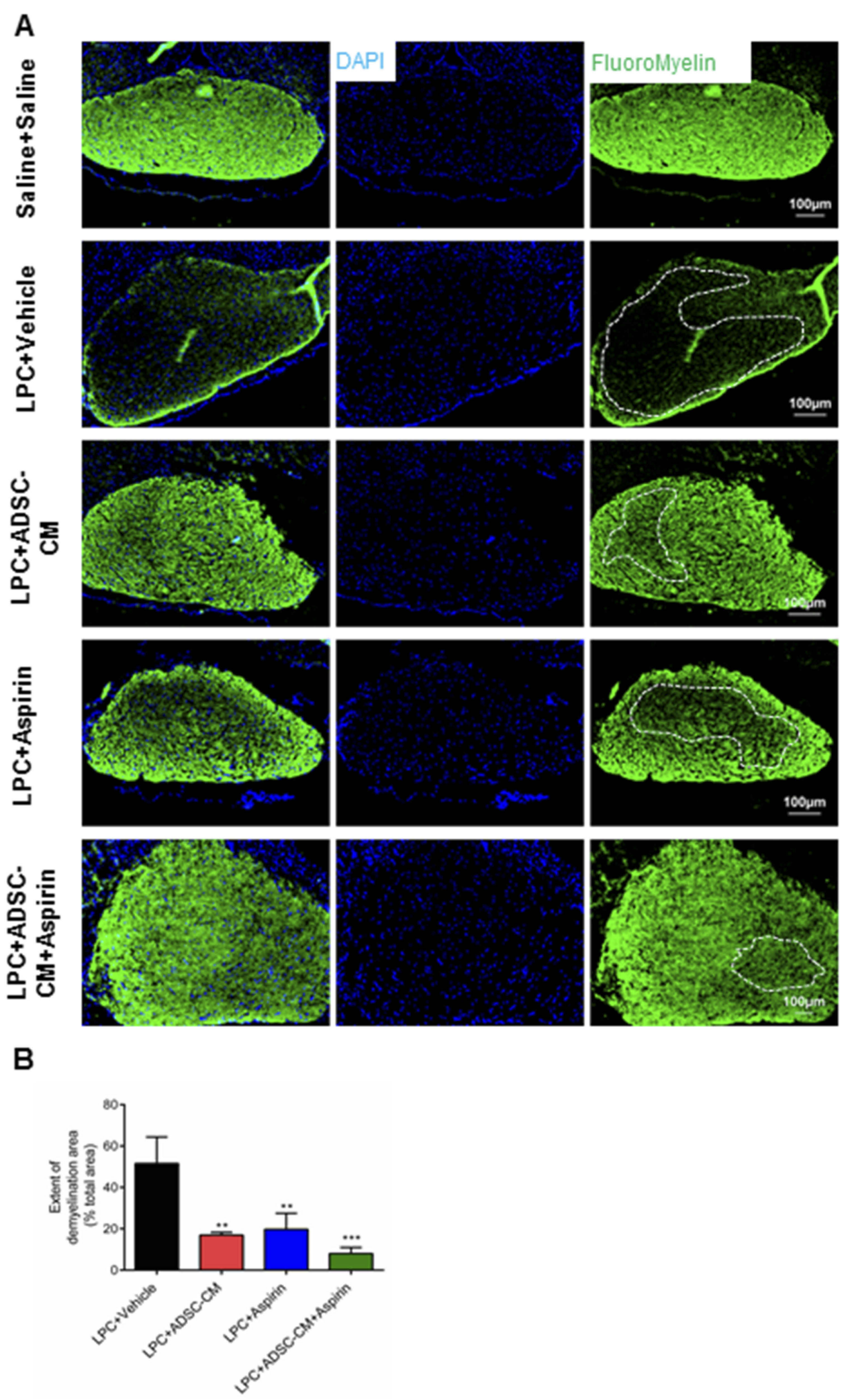

Figure 3 Effect of ADSCs-CM and aspirin on demyelination levels of the optic chiasm. (A) FluoroMyelin staining of optic chiasm's sections on day I4 post LPC injection. DAPI: nucleus stain. Scale bar: $100 \mu \mathrm{m}$. (B) ADSCs-CM, aspirin or combination of ADSCs-CM and aspirin decreased the extent of demyelination areas. One-way ANOVA followed by Tukey post-test were used for statistical analysis. $* * P<0.01$ and $* * * P<0.001$ compared to $L P C+$ vehicle. $n=3$. 


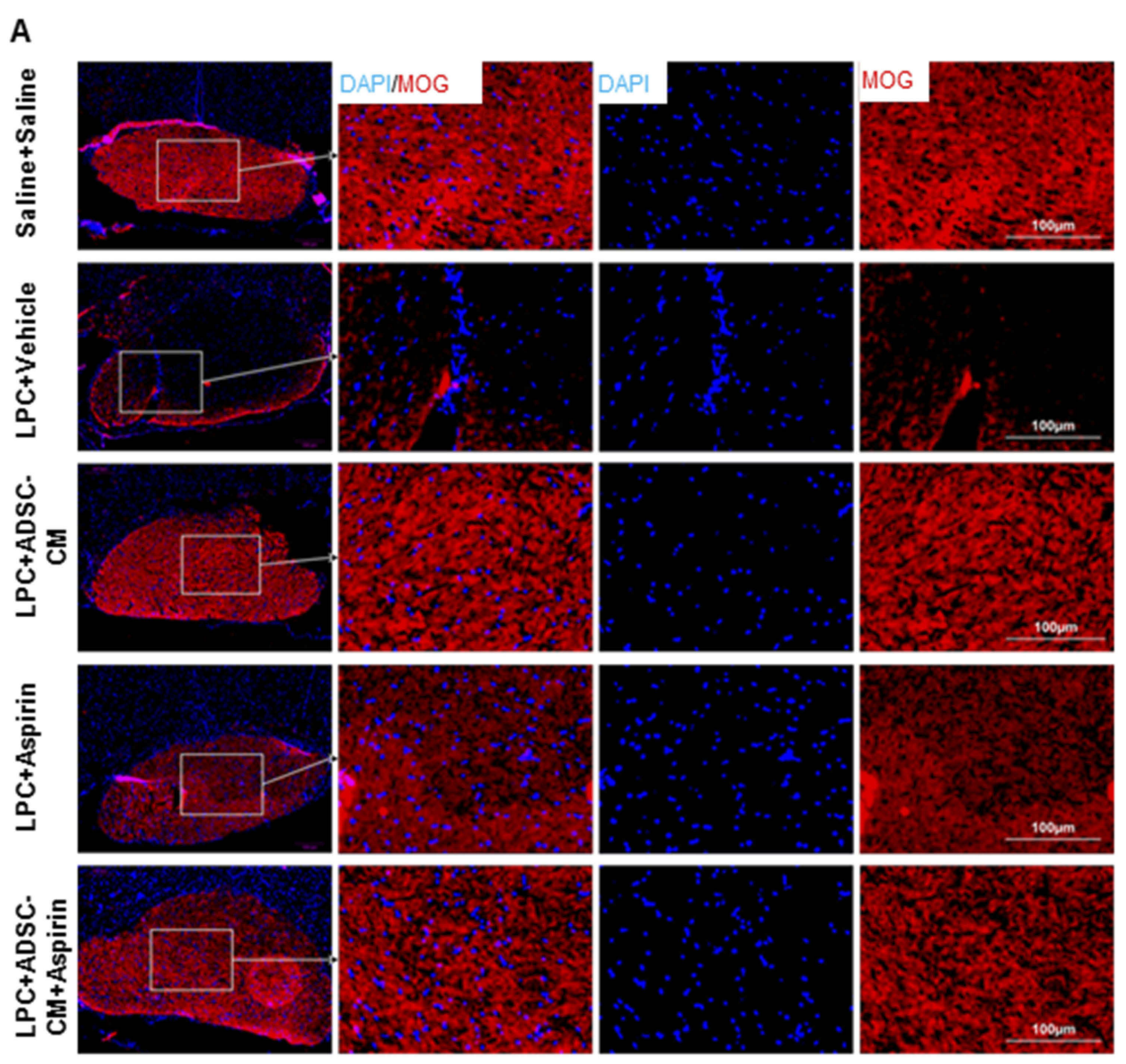

B

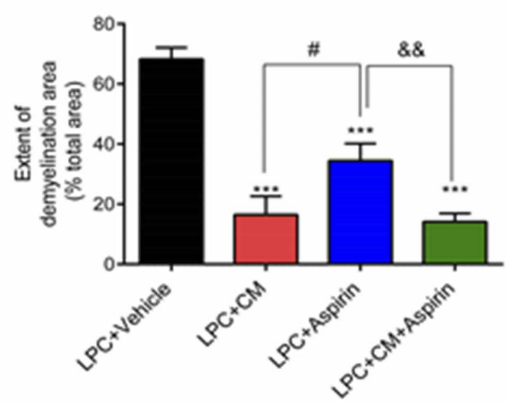

Figure 4 Effect of ADSCs-CM and aspirin on extent of demyelination areas. (A) Immunostaining against MOG as mature myelin marker on day 14 post insult. DAPI: nuclei stain, scale bar: $100 \mu \mathrm{m}$. (B) Quantification of MOG staining showed that administration of ADSCs-CM, aspirin or ADSCs-CM + aspirin alleviated the level of demyelination. One-way ANOVA followed by Tukey post-test were used for statistical analysis. $* * * P<0.001$ compared to the LPC + vehicle; ${ }^{*}<<0.05$ compared to the LPC + ADSCs-CM and ${ }^{\& \&} P<0.01$ compared to the LPC + aspirin. $n=3$.

was also significantly enhanced in animals treated with both ADSCs-CM and aspirin compared to the LPC + vehicle $(P<0.001)$. In addition, there was a significant difference in PDGFR $\alpha$ expressing cells between LPC + ADSCs-CM + aspirin and LPC + ADSCs-CM $(P<0.01)$ or $\mathrm{LPC}+\operatorname{aspirin}(P<0.01)$ (Figure $5 \mathrm{~A}$ and $\mathrm{B})$.

\section{Co-administration of ADSCs-CM and} aspirin ameliorated astrocyte activation

To examine the effect of ADSCs-CM and aspirin on astrocytes' activation, immunostaining against GFAP as astrocyte marker was performed in experimental groups. The number of GFAP expressing cells significantly 

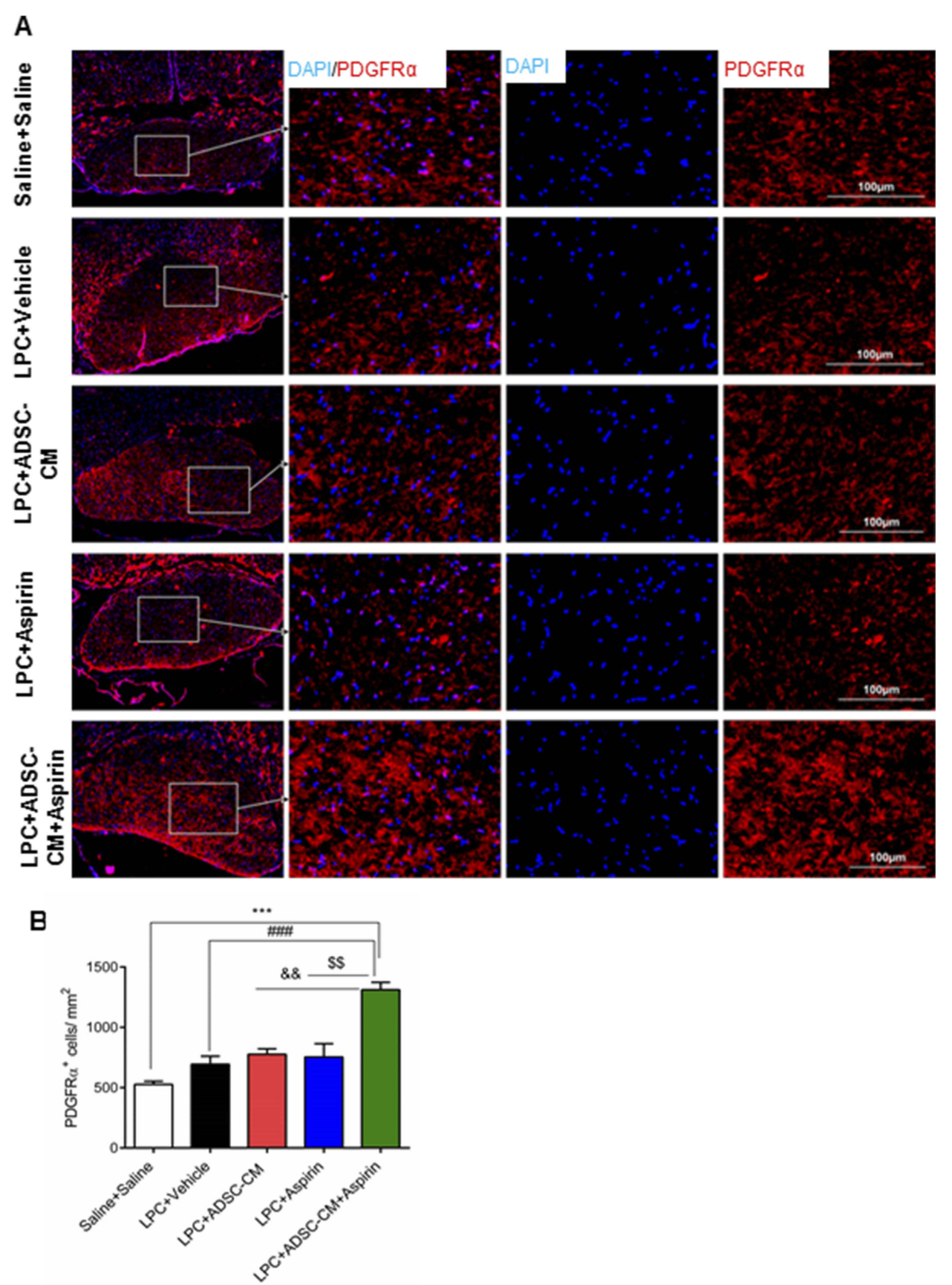

Figure 5 Effect of ADSCs-CM and aspirin on the number of oligodendrocyte precursor cells. (A) Immunostaining against PDGFR $\boldsymbol{\alpha}$ as oligodendrocyte precursor cells marker on brain sections. DAPI: nuclei stain. Scale bar: $100 \mu \mathrm{m}$. (B) Quantification of immunostaining data showed that co-administration of ADSCs-CM and aspirin increased the number of PDGFRa expressing cells following LPC injection. One-way ANOVA followed by Tukey post-test were used for statistical analysis. ${ }_{* * *} P<0.001$ compared to the saline + saline; $P<0.001$ compared to the $\mathrm{LPC}+$ vehicle; ${ }^{\& \&} P<0.01$ compared to the LPC + ADSCs-CM and ${ }^{\$} P<0.01$ compared to the $\mathrm{LPC}+$ aspirin. $\mathrm{n}=3$.

increased in LPC + vehicle receiving animals compared to the saline + saline $(P<0.001)$. Interestingly, ADSCs$\mathrm{CM}$, aspirin or ADSCs-CM + aspirin significantly alleviated the level of astrocyte activation compared to the LPC + vehicle $(P<0.001)$. Additionally, co-administration of ADSCs-CM and aspirin effectively reduced the level of astrocyte activation in the optic chiasm compared to the $\mathrm{LPC}+$ aspirin $(P<0.05)$ (Figure 6A and B). These data indicated that co-administration of ADSCs$\mathrm{CM}$ and aspirin considerably alleviated the level of astrocytes' activation in LPC-induced demyelination model. 
A
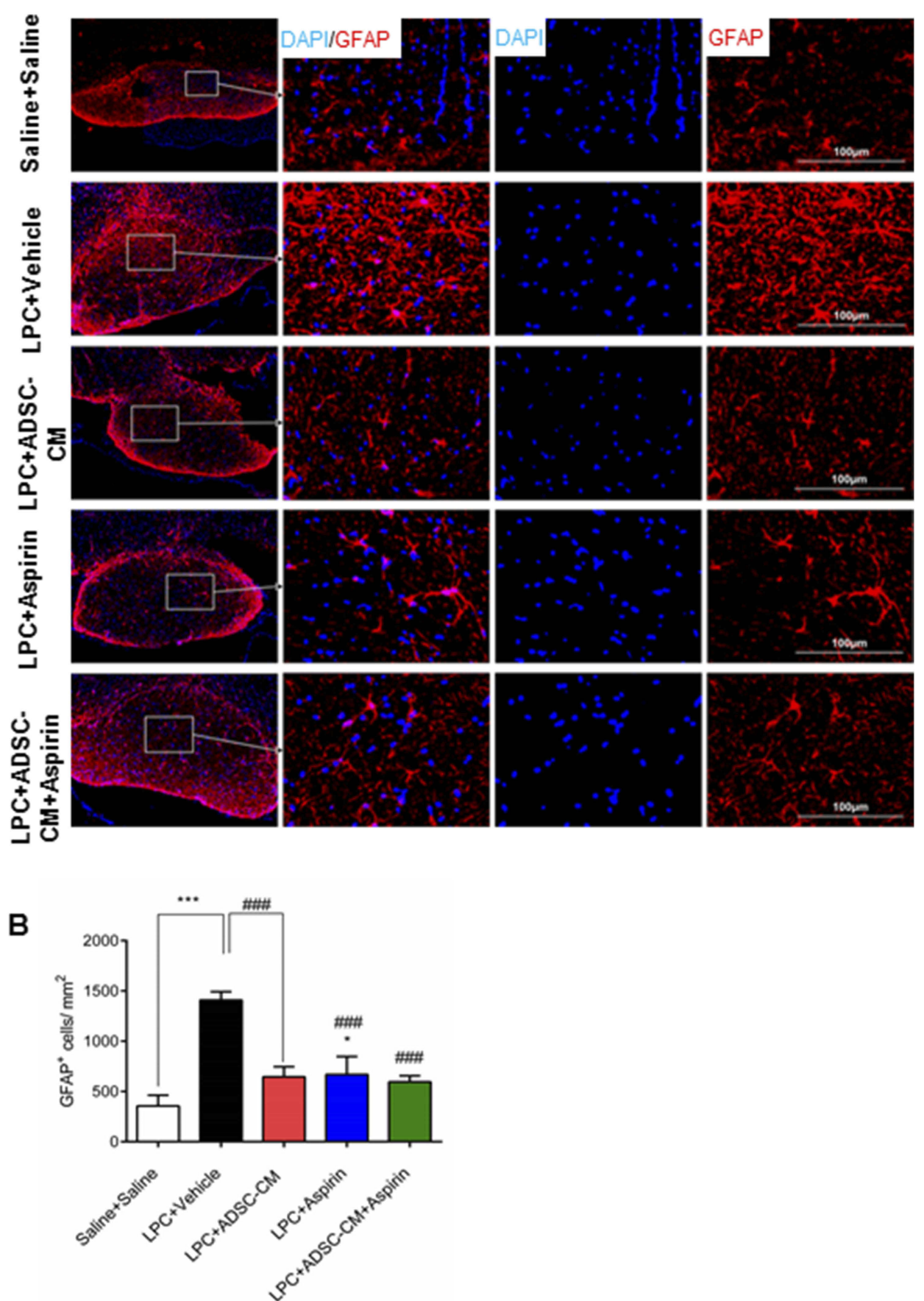

Figure 6 Effect of ADSCs-CM and aspirin on astrocyte activation of optic chiasm in LPC-induced demyelination model. (A) Immunostaining against GFAP as astrocyte marker on day 14 post LPC injection. DAPI: nuclei stain. Scale bar: $100 \mu \mathrm{m}$. (B) ADSCs-CM, aspirin or ADSCs-CM + aspirin ameliorated the number of GFAP positive cells in the demyelination context. One-way ANOVA followed by Tukey post-test were used for statistical analysis. $* P<0.05$ and $* * * P<0.001$ compared to the saline + saline and $P<0.001$ compared to the $L P C+$ vehicle. $n=3$.

\section{Discussion}

In recent years, development of new therapies for enhancement of endogenous remyelination has attracted considerable attention. ${ }^{45,46}$ Here, we demonstrated that co-administration of ADSCs-CM and aspirin improved the functional recovery of optic pathway following LPC administration. Furthermore, the levels of demyelination and astrocytes' activation were reduced in the optic chiasm. A significant increase in population of OPCs was also observed in animals under treatment of both ADSCs-CM and aspirin. 
Several pieces of evidence suggested that aspirin and ADSCs-CM, apart from their immunomodulatory and anti-inflammatory effects, play an important role in promotion of endogenous remyelination. In line with previous reports, ${ }^{33,34,36,39,47}$ we also showed that local injection of LPC into the rat optic chiasm reduces the conductivity of visual signals and co-administration of ADSCs-CM and aspirin could significantly decrease the latency of VEP waves.

To explore the possible underlying mechanism/s of aspirin and ADSCs-CM in improvement of functional recovery of optic pathway, the extent of demyelination was evaluated in context of local demyelination. The results demonstrated the level of demyelination was especially reduced in animals which were treated with ADSCs-CM and aspirin. In agreement with our data, a previous report also showed that aspirin treatment, by increasing the levels of CNTF as pro-myelinating trophic factor, increases myelin-associated proteins in oligodendrocytes and improves the oligodendrocyte protection in TNF- $\alpha$-induced insult. ${ }^{30}$ In an interesting study by Chen et al, it has been demonstrated that treatment with aspirin improves cognitive performance and enhances remyelination process after white matter lesion. ${ }^{7}$ To determine the underlying mechanism of aspirin in promotion of OPCs' differentiation, Huang et al's report showed that aspirin can directly target oligodendroglial lineage cells and enhances the OPCs' differentiation via inhibition of $\mathrm{Wnt} / \beta$-catenin signaling pathway. ${ }^{29}$ Jadasz et al illustrated that MSC-CM elevates the expression levels of myelin markers and promotes endogenous myelin repair. ${ }^{5}$ It has also been shown that transplantation of bone marrowderived MSCs into the lateral ventricles increases myelin content through secretion of soluble factors into cerebral spinal fluid (CSF) and enhancement of endogenous oligodendrogenic potential of the subventricular zone (SVZ). ${ }^{48}$ Additionally, it has been suggested that $\mathrm{CM}$ from bone marrow-derived MSCs significantly reduces the neuronal apoptosis and lesion area in spinal cord injury model in rats. ${ }^{20}$ Conditioned media from the stem cells of human exfoliated deciduous teeth (SHED-CM) also reduces the severity of EAE symptoms and attenuates the levels of demyelination and axonal injury. ${ }^{38}$ Hao et al's report also indicated that ADSCs-CM enhances neuroprotection by inhibiting neuronal apoptosis and increasing nerve regeneration in cultured neurons. ${ }^{49}$ In parallel with previously mentioned evidence, findings of the present study also demonstrated that aspirin or ADSCs-CM significantly enhances the myelin protection in local demyelination context. However, a stronger myelin preserving effect was observed when aspirin and ADSCs-CM were simultaneously administrated. These data may be a result of the synergic effects of aspirin and ADSC-CM on enhancement of endogenous remyelination.

In the next part of the experiment, we explored the possible effect of aspirin and ADSCs-CM on population of OPCs. Our data indicated that co-administration of aspirin and ADSCs-CM remarkably increased the number of OPCs, while administration of aspirin or ADSCs-CM alone did not lead to a significant increase in population of OPCs. A previous report by Chen et al demonstrated that in vivo treatment with low dose of aspirin $(25 \mathrm{mg} / \mathrm{kg})$ significantly elevates the number of OPCs while administration of aspirin at relatively high doses $(100-200 \mathrm{mg} / \mathrm{kg}$ ) increases the number of mature oligodendrocytes. ${ }^{7}$ Similarly, an in vitro study also indicated that low and high doses of aspirin promote the proliferation and differentiation of cultured cells, respectively. ${ }^{7}$ In contrast to Chen et al's report, in the present study, a significant increase in the number of PDGFR $\alpha$ positive cells was not found when aspirin or ADSCs-CM was administrated alone. In line with our results, a study by Huang et al also suggested that aspirin enhances endogenous repair, mainly by promoting oligodendrocytes' differentiation, and has no effect on OPCs' proliferation even in lower dosage. ${ }^{29}$ Additionally, it has been shown that aspirin promotes OPCs' differentiation through increasing CNTF levels in cultured astrocytes. ${ }^{30}$ Moreover, Jadasz et al demonstrated that MSC-CM accelerated OPC differentiation even under astrocytic endorsing conditions. ${ }^{5}$ The discrepancies between our data and Chen et al's report may be a result from different experimental protocols and settings.

Although successful remyelination is very dependent on recruitment, activation, and differentiation of OPCs, ${ }^{5}$ excessive glial activation and release of numerous inflammatory factors from activated astrocytes and microglia in the damaged areas remarkably hamper the overall degree of efficient remyelination in the CNS. ${ }^{50}$ To this end, we analyzed the level of astrocytes' activation and our findings showed that treatment with aspirin, ADSCs-CM or aspirin + ADSCs-CM significantly alleviated the number of GFAP positive cells following LPC injection. In agreement with our results, a previous study by Jadasz et al also demonstrated that ADSCs-CM significantly reduces the levels of GFAP expressing cells. ${ }^{5}$ In addition, it has been shown that ADSCs-CM decreases the expression of inflammatory factors in an EAE model. ${ }^{2} \mathrm{Li}$ et al's report indicated that ADSCs-CM remarkably attenuates the serum level of TNF- $\alpha$, IL-1, and IL-6 
in lipopolysaccharide (LPS) treated mice as well as cultured macrophages. ${ }^{51}$ In addition, both AMSC and ADSCs-CM significantly reduced the clinical symptoms and disease activity index of colitis in mice. ${ }^{52}$ Furthermore, an increased level of IL10 and decreased level of IL-17 were found after treatment with ADSCs or ADSCs-CM. ${ }^{52}$ It has been shown that a single injection of SHED-CM reduces the infiltration of inflammatory cells and expression of pro-inflammatory cytokines in the spinal cord of EAE mice. ${ }^{38}$ Administration of MSC-CM also significantly attenuates the expression of TNF- $\alpha$, IL- 6 , and inducible NO synthase (iNOS) both in transgenic and non-transgenic astrocytes. ${ }^{53}$ It has been demonstrated that MSC-CM increases cell proliferation and reduces the apoptosis and expression of inflammatory factors from human gingival fibroblasts which were stimulated by LPS. ${ }^{54}$ Yao et al's study suggested that aspirin-triggered lipoxin A4 inhibits the expression of iNOS and cyclooxygenase 2 (COX2) in astrocytes via NF-kB-dependent pathway. ${ }^{50}$ Most recently, Mondal et al showed that treatment with low dose of aspirin suppresses the clinical symptoms of EAE and reduces the expression level of inflammatory factors. ${ }^{3}$ Pettit et al also illustrated that a combination of aspirin and docosahexaenoic acid led to augmentation in total glutathione levels as well as a reduction in expression levels of IL-6, TNF- $\alpha$, and NO in activated microglia. ${ }^{55}$ Furthermore, it has been shown that aspirin attenuates the mRNA and protein levels of GFAP by targeting NF- $\mathrm{kB}{ }^{56}$

In conclusion, our findings provide the first evidence that co-administration of aspirin and ADSCs-CM can improve the functional recovery of optic pathway by at least their myelin preserving activity and inhibitory effect on astrocytes' activation. However, further studies are needed to elucidate the precise mechanism/s of aspirin and ADSCs-CM in enhancement of endogenous myelin repair in context of demyelination.

\section{Abbreviations}

MS, multiple sclerosis; CNS, central nervous system; OPCs, oligodendrocyte precursor cells; MSCs, mesenchymal stem cells; NSCs, neural stem cells; ADSC-CM, adipose tissuederived stem cells conditioned media; EAE, experimental autoimmune encephalomyelitis; ASA, acetylsalicylic acid; CNTF, ciliary neurotrophic factor; LPC, lysolecithin; VEP, visual evoked potential; i.p., intraperitoneal; PBS, phosphate buffered saline; PFA, paraformaldehyde; NGS, normal goat serum; GFAP, glial fibrillary acidic protein; PDGFR $\alpha$, plateletderived growth factor receptor $\alpha$; MOG, myelin-oligodendrocyte glycoprotein; DAPI: 4', 6-diamidino-2-phenylindole; CSF, cerebral spinal fluid; SVZ, subventricular zone; LPS, lipopolysaccharide; SHED, stem cells of human exfoliated deciduous teeth; iNOS, inducible NO synthase; COX2, Cyclooxygenase 2.

\section{Acknowledgment}

This work was supported by a grant from the Deputy of Research and Technology (no. 9603710), Babol University of Medical Sciences, Babol, Iran and was performed as part of Master of Science thesis in Anatomical Sciences. The authors would like to acknowledge the kind assistance and support of Temad Company (Karaj, Iran) for providing aspirin and Prof. Mohammad Javan (Department of Physiology, Tarbiat Modares University, Tehran, Iran).

\section{Disclosure}

The authors declare no conflict of interest related to this study.

\section{References}

1. Ghasemi-Kasman M, Zare L, Baharvand H, Javan M. In vivo conversion of astrocytes to myelinating cells by miR-302/367 and valproate to enhance myelin repair. J Tissue Eng Regen Med. 2018;12(1):e462-e472. doi:10.1002/term.2276

2. Yousefi F, Ebtekar M, Soudi S, Soleimani M, Hashemi SM. In vivo immunomodulatory effects of adipose-derived mesenchymal stem cells conditioned medium in experimental autoimmune encephalomyelitis. Immunol Lett. 2016;172:94-105. doi:10.1016/j.imlet.2016.02.016

3. Mondal S, Jana M, Dasarathi S, Roy A, Pahan K. Aspirin ameliorates experimental autoimmune encephalomyelitis through interleukin-11mediated protection of regulatory T cells. Sci Signal. 2018;11(558): eaar8278. doi:10.1126/scisignal.aar8278

4. Mulakayala N, Rao P, Iqbal J, Bandichhor R, Oruganti S. Synthesis of novel therapeutic agents for the treatment of multiple sclerosis: a brief overview. Eur J Med Chem. 2013;60:170-186. doi:10.1016/j. ejmech.2012.10.055

5. Jadasz JJ, Kremer D, Göttle P, et al. Mesenchymal stem cell conditioning promotes rat oligodendroglial cell maturation. PLoS One. 2013;8(8):e71814. doi:10.1371/journal.pone.0071814

6. Kotter MR, Stadelmann C, Hartung H-P. Enhancing remyelination in disease - can we wrap it up? Brain. 2011;134(7):1882-1900. doi:10. 1093/brain/awr014

7. Chen J, Zuo S, Wang J, et al. Aspirin promotes oligodendrocyte precursor cell proliferation and differentiation after white matter lesion. Front Aging Neurosci. 2014;6:7. doi:10.3389/fnagi.2014.00007

8. Zafranskaya MM, Nizheharodova DB, Yurkevich MY, et al. In vitro assessment of mesenchymal stem cells immunosuppressive potential in multiple sclerosis patients. Immunol Lett. 2013;149(1-2):9-18. doi:10.1016/j.imlet.2012.10.010

9. Dazzi F, Krampera M. Mesenchymal stem cells and autoimmune diseases. Best Pract Res Clin Haematol. 2011;24(1):49-57. doi:10.1016/j.beha.2011.01.002

10. Cuascut FX, Hutton GJ. Stem cell-based therapies for multiple sclerosis: current perspectives. Biomedicines. 2019;7(2):26. doi:10.33 90/biomedicines 7020026

11. Uccelli A, Moretta L, Pistoia V. Mesenchymal stem cells in health and disease. Nature Rev Immunol. 2008;8(9):726. doi:10.1038/nri2395

12. Davoodian N, Lotfi AS, Soleimani M, Mowla SJ. MicroRNA-122 overexpression promotes hepatic differentiation of human adipose tissue-derived stem cells. J Cell Biochem. 2014;115(9):1582-1593. doi: $10.1002 /$ jcb. 24822 
13. Lin F. Adipose tissue-derived mesenchymal stem cells: a fat chance of curing kidney disease? Kidney Int. 2012;82(7):731-733. doi:10.1038/ki.2012.158

14. Zuk P. Adipose-derived stem cells in tissue regeneration: a review. ISRN Stem Cells. 2013;2013:1-35.

15. Aggarwal S, Pittenger MF. Human mesenchymal stem cells modulate allogeneic immune cell responses. Blood. 2005;105(4):1815-1822. doi:10.1182/blood-2004-04-1559

16. Uccelli A, Benvenuto F, Laroni A, Giunti D. Neuroprotective features of mesenchymal stem cells. Best Pract Res Clin Haematol. 2011;24 (1):59-64. doi:10.1016/j.beha.2011.01.004

17. Mammana S, Gugliandolo A, Cavalli E, et al. Human Gingival Mesenchymal Stem Cells (GMSCs) pre-treated with vesicular Moringin nanostructures as a new therapeutic approach in a mouse model of spinal cord injury. J Tissue Eng Regen Med. 2019. doi:10.1002/term. 2857

18. Rivera FJ, Couillard-Despres S, Pedre X, et al. Mesenchymal stem cells instruct oligodendrogenic fate decision on adult neural stem cells. Stem Cells. 2006;24(10):2209-2219. doi:10.1634/stemcells.2005-0614

19. Bai L, Lennon DP, Eaton V, et al. Human bone marrow-derived mesenchymal stem cells induce Th2-polarized immune response and promote endogenous repair in animal models of multiple sclerosis. Glia. 2009;57(11):1192-1203. doi:10.1002/glia.20841

20. Cantinieaux D, Quertainmont R, Blacher S, et al. Conditioned medium from bone marrow-derived mesenchymal stem cells improves recovery after spinal cord injury in rats: an original strategy to avoid cell transplantation. PLoS One. 2013;8(8):e69515. doi:10.1371/journal.pone.0069515

21. Pawitan JA. Prospect of stem cell conditioned medium in regenerative medicine. Biomed Res Int. 2014;2014:1-14.

22. Noverina R, Widowati W, Ayuningtyas W, et al. Growth factors profile in conditioned medium human adipose tissue-derived mesenchymal stem cells (CM-hATMSCs). Clin Nutr Exp. 2019;24:34-44. doi:10.1016/j.yclnex.2019.01.002

23. Wei X, Zhao L, Zhong J, et al. Adipose stromal cells-secreted neuroprotective media against neuronal apoptosis. Neurosci Lett. 2009;462(1):76-79. doi:10.1016/j.neulet.2009.06.054

24. Wilkins A, Kemp K, Ginty M, Hares K, Mallam E, Scolding N. Human bone marrow-derived mesenchymal stem cells secrete brainderived neurotrophic factor which promotes neuronal survival in vitro. Stem Cell Res. 2009;3(1):63-70. doi:10.1016/j.scr.2009.02.006

25. Yazdi A, Khanghahi AM, Baharvand H, Javan M. Fingolimod enhances oligodendrocyte differentiation of transplanted human induced pluripotent stem cell-derived neural progenitors. Iran $J$ Pharm Res. 2018;17(4):1444.

26. Shaygannejad V, Janghorbani M, Ashtari F, Zakeri H. Comparison of the effect of aspirin and amantadine for the treatment of fatigue in multiple sclerosis: a randomized, blinded, crossover study. Neurol Res. 2012;34(9):854-858. doi:10.1179/1743132812Y.0000000081

27. Wingerchuk DM, Benarroch E, O'Brien P, et al. A randomized controlled crossover trial of aspirin for fatigue in multiple sclerosis. Neurology. 2005;64(7):1267-1269. doi:10.1212/01.WNL.0000156803.23698.9A

28. Tsau S, Emerson MR, Lynch SG, LeVine SM. Aspirin and multiple sclerosis. BMC Med. 2015;13(1):153. doi:10.1186/s12916-015-0394-4

29. Huang N, Chen D, Wu X, et al. Aspirin promotes oligodendroglial differentiation through inhibition of Wnt signaling pathway. Mol Neurobiol. 2016;53(5):3258-3266. doi:10.1007/s12035-015-9241-z

30. Modi KK, Sendtner M, Pahan K. Up-regulation of ciliary neurotrophic factor in astrocytes by aspirin implications for remyelination in multiple sclerosis. J Biol Chem. 2013;288(25):18533-18545. doi:10.1074/jbc.M112.447268

31. Louis J-C, Magal E, Takayama S, Varon S. CNTF protection of oligodendrocytes against natural and tumor necrosis factor-induced death. Science. 1993;259(5095):689-692. doi:10.1126/science.8430320

32. Plemel JR, Michaels NJ, Weishaupt N, et al. Mechanisms of lysophosphatidylcholine-induced demyelination: a primary lipid disrupting myelinopathy. Glia. 2018;66(2):327-347. doi:10.1002/glia.23245
33. Sherafat MA, Javan M, Mozafari S, Mirnajafi-Zadeh J, Motamedi F. Castration attenuates myelin repair following lysolecithin induced demyelination in rat optic chiasm: an evaluation using visual evoked potential, marker genes expression and myelin staining. Neurochem Res. 2011;36(10):1887. doi:10.1007/s11064-011-0501-7

34. Baradaran S, Moghaddam AH, Ghasemi-Kasman M. Hesperetin reduces myelin damage and ameliorates glial activation in lysolecithin-induced focal demyelination model of rat optic chiasm. Life Sci. 2018;207:471-479. doi:10.1016/j.1fs.2018.07.001

35. You Y, Gupta VK, Chitranshi N, Reedman B, Klistorner A, Graham SL. Visual evoked potential recording in a rat model of experimental optic nerve demyelination. JoVE. 2015;29(101):e52934.

36. Naeimi R, Baradaran S, Ashrafpour M, Moghadamnia AA, GhasemiKasman M. Querectin improves myelin repair of optic chiasm in lyolecithin-induced focal demyelination model. Biomed Pharmacother. 2018;101:485-493. doi:10.1016/j.biopha.2018.02.125

37. Azandaryani ZS, Davoodian N, Samiei A, Rouzbehan S. Insulin-like growth factor-I promotes hepatic differentiation of human adipose tissue-derived stem cells. Cell Biol Int. 2019;43(5):476-485.

38. Shimojima C, Takeuchi H, Jin S, et al. Conditioned medium from the stem cells of human exfoliated deciduous teeth ameliorates experimental autoimmune encephalomyelitis. $J$ Immunol. 2016;196 (10):4164-4171. doi:10.4049/jimmunol.1501457

39. Daneshdoust D, Khalili-Fomeshi M, Ghasemi-Kasman M, et al. Pregabalin enhances myelin repair and attenuates glial activation in lysolecithin-induced demyelination model of rat optic chiasm. Neuroscience. 2017;344:148-156. doi:10.1016/j.neuroscience.2016. 12.037

40. Hashemian M, Ghasemi-Kasman M, Parsian H, Sadeghi F. Fingolimod (FTY720) improves the functional recovery and myelin preservation of the optic pathway in focal demyelination model of rat optic chiasm. Brain Res Bull. 2019. doi:10.1016/j.brainresbull.2019.08.014

41. Naeimi R, Safarpour F, Hashemian M, et al. Curcumin-loaded nanoparticles ameliorate glial activation and improve myelin repair in lysolecithininduced focal demyelination model of rat corpus callosum. Neuroscience letters. 2018;674:1-10.

42. Ahmadian SR, Ghasemi-Kasman M, Pouramir M, Sadeghi F. Arbutin attenuates cognitive impairment and inflammatory response in pentylenetetrazol-induced kindling model of epilepsy. Neuropharmacology. 2019;146(1):117-127.

43. Gol M, Ghorbanian D, Hassanzadeh S, Javan M, Mirnajafi-Zadeh J, Ghasemi-Kasman M. Fingolimod enhances myelin repair of hippocampus in pentylenetetrazol-induced kindling model. Eur J Pharm Sci. 2017;96:72-83. doi:10.1016/j.ejps.2016.09.016

44. Akbari A, Khalili-Fomeshi M, Ashrafpour M, Moghadamnia AA, Ghasemi-Kasman M. Adenosine A2A receptor blockade attenuates spatial memory deficit and extent of demyelination areas in lyolecithin-induced demyelination model. Life Sci. 2018;205:63-72. doi:10.1016/j.1fs.2018.05.007

45. Fancy SP, Kotter MR, Harrington EP, et al. Overcoming remyelination failure in multiple sclerosis and other myelin disorders. Exp Neurol. 2010;225(1):18-23. doi:10.1016/j.expneurol.2009.12.020

46. Huang JK, Fancy SP, Zhao C, Rowitch DH, Franklin RJ. Myelin regeneration in multiple sclerosis: targeting endogenous stem cells. Neurotherapeutics. 2011;8(4):650-658. doi:10.1007/s13311-011-0065-x

47. Mozafari S, Sherafat MA, Javan M, Mirnajafi-Zadeh J, Tiraihi T. Visual evoked potentials and MBP gene expression imply endogenous myelin repair in adult rat optic nerve and chiasm following local lysolecithin induced demyelination. Brain Res. 2010;1351:50-56. doi:10.1016/j.brainres.2010.07.026

48. Cruz-Martinez P, González-Granero S, Molina-Navarro M, et al. Intraventricular injections of mesenchymal stem cells activate endogenous functional remyelination in a chronic demyelinating murine model. Cell Death Dis. 2016;7(5):e2223. doi:10.1038/ cddis. 2016.130 
49. Hao P, Liang Z, Piao H, et al. Conditioned medium of human adipose-derived mesenchymal stem cells mediates protection in neurons following glutamate excitotoxicity by regulating energy metabolism and GAP-43 expression. Metab Brain Dis. 2014;29(1):193205. doi:10.1007/s11011-014-9490-y

50. Yao C, Yang D, Wan Z, et al. Aspirin-triggered lipoxin A4 attenuates lipopolysaccharide induced inflammatory response in primary astrocytes. Int Immunopharmacol. 2014;18(1):85-89. doi:10.1016/j. intimp.2013.10.028

51. Li Y, Gao X, Wang J. Human adipose-derived mesenchymal stem cell-conditioned media suppresses inflammatory bone loss in a lipopolysaccharide-induced murine model. Exp Ther Med. 2018;15 (2):1839-1846. doi:10.3892/etm.2017.5606

52. Heidari M, Pouya S, Baghaei K, et al. The immunomodulatory effects of adipose-derived mesenchymal stem cells and mesenchymal stem cells-conditioned medium in chronic colitis. J Cell Physiol. 2018;233(11):8754-8766. doi:10.1002/jcp.26765
53. Sun H, Bénardais K, Stanslowsky N, et al. Therapeutic potential of mesenchymal stromal cells and MSC conditioned medium in amyotrophic lateral sclerosis (ALS)-in vitro evidence from primary motor neuron cultures, NSC-34 cells, astrocytes and microglia. PLoS One. 2013;8(9):e72926. doi:10.1371/journal.pone.0072926

54. Qie H, Fan S, Feng Z, Wang F, Hou X, Shan L. The inhibitory effect of mesenchymal stem cell conditioned medium on the secretion of inflammatory factor of human gingival fibroblast induced by lipopolysaccharide. Int J Clin Exp Med. 2018;11(6):5715-5721.

55. Pettit LK, Varsanyi C, Tadros J, Vassiliou E. Modulating the inflammatory properties of activated microglia with docosahexaenoic acid and aspirin. Lipids Health Dis. 2013;12(1):16. doi:10.1186/1476511X-12-16

56. Bae M-K, Kim S-R, Lee H-J, et al. Aspirin-induced blockade of NF$\kappa \mathrm{B}$ activity restrains up-regulation of glial fibrillary acidic protein in human astroglial cells. Biochim Et Biophys Acta (bba)-mol Cell Res. 2006;1763(3):282-289. doi:10.1016/j.bbamcr.2006.01.005

\section{Publish your work in this journal}

Neuropsychiatric Disease and Treatment is an international, peerreviewed journal of clinical therapeutics and pharmacology focusing on concise rapid reporting of clinical or pre-clinical studies on a range of neuropsychiatric and neurological disorders. This journal is indexed on PubMed Central, the 'PsycINFO' database and CAS, and is the official journal of The International Neuropsychiatric Association (INA). The manuscript management system is completely online and includes a very quick and fair peer-review system, which is all easy to use. Visit http://www.dovepress.com/testimonials.php to read real quotes from published authors. 